\title{
Risk Factors and Outcome of Preterm Labour in Tertiary Health Centre
}

\author{
MST. AFROZA KHANUM ${ }^{1}$, SALMALAVEREEN ${ }^{2}$, MONIRUZZAMAN $^{3}$, ROMANA $^{4}$
}

\begin{abstract}
:
Background: Currently preterm labour is one of the most challenging problems confronting the obstetricians and perinatologists. This unfortunate episode accounts for $50-75 \%$ of the perinatal mortality.

Methods: A cross sectional study was conducted on 210 pregnant women with preterm labour admitted in Monno Medical College Hospital, Manikganj from June 2014 to December 2015, to study the causes and outcome of preterm birth in Tertiary health centre of Manikganj.

Results: Occurence of preterm birth was $13.82 \%$; 47.14\% occured between 34-37 weeks of gestation; $33.80 \%$ occured 31-33 weeks of gestation and occurred in 28-30 weeks $19.04 \%$. About $22 \%$ patients presenting with preterm labour had a past history of abortions and $14.3 \%$ had a history of preterm delivery. Premature rupture of membranes was found to be the most common risk factor related with preterm labour in the present pregnancy. Genitourinary tract infection was the next important risk factor of preterm labour; $24.8 \%$ (86) patients had either vaginal infection (19.5\%) or urinary infection $(21.4 \%)$ or both. Another important risk factor identified in this study was antepartum haemorrage which was cause in $11.4 \%$ cases. Preterm babies commonly suffered from various complications like jaundice (32.1\%), respiratory distress syndrome (22.6\%), asphyxia (13.5\%), sepsis, hypoglycemia and coagulopathy.

Conclusion: Most of the preterm births occured between 34-37 weeks of gestation. Most common risk factors of preterm births are history of abortion and preterm delivery in previous pregnancy; PROM UTI vaginal infection, PIH and APH in correct pregnancy. Newborn jaundice, $R D S$ and birth asphyxia are the common neonatal morbidity in preterm labour. Identifying risk factors to prevent the onset of preterm labour and advanced neonatal care unit can help decrease neonatal morbidity and mortality.
\end{abstract}

Key Words: Preterm labour, Risk factor, Neonatal outcome.

\section{Introduction:}

Preterm labour is one of the most challenging problems faced by obstetricians and perinatologist because of women's pregnancy takes a heavy toll of high perinatal mortality and morbidity. In developing country among the total 27 million babies born annually, 3.6 million babies are born preterm and over 300,000 of these preterm babies die each year because of associated complication. ${ }^{1}$
It is estimated that the risks of neonatal mortality and low birth weight are increased by almost $50 \%$ if maternal age at childbirth is less than 20 years. $^{2}$

A very strong association has been found with education status and socio-economic status in the cases of preterm births. Despite the advances in fetomaternal medicine the preterm rate continues to be high. Women's education is associated with decline in preterm birth rate. ${ }^{3}$

1. Assistant Professor,(Obst.\&Gynae), Monno Medical College.

2. Assistant Professor,(Obst\&Gynae), IbnSina Medical College.

3. Assistant Professor, Pediatric Dept., Monno Medical College.

4. R.S.(Gynae), Monno Medical College.

Address of Correspondence: Dr. Mst. AfrozaKhanum, DGO,AssistantProfessor,Obs\&Gynae,Monno Medical College. Email:dr.afrozakhanum63@gmail.com, Mobile-01711269190 
Recent studies have shown association between preterm birth and stress and depression, negative life events, perception of racial discrimination and domestic violence are also associated with higher preterm births. ${ }^{4}$

A significant proportion of preterm births are preventable by adequate antenatal care, female literacy and health education, which increase the awareness about antenatal care. So our study aims to find out the main risk factors related to preterm births specially those which can be intervened upon so that proper measure can be undertaken to decrease the preterm birth rate and neonatal morbidity and mortality associated with prematurity.

\section{Materials And Methods:}

This cross sectional study was carried out in the Department of Obstetrics and gynecology of Monno Medical College Hospital, Manikganj, on 210 pregnant women with preterm labour 28-34+6 week of gestation from June 2014 to December 2015.

Patients enrolled into study were subjected to a detailed history taking with respect to age, parity, previous pregnancy outcomes and for the presence of any risk factors in this pregnancy including GDM, $\mathrm{PIH}$, anaemia or any other medical disease. A thorough general physical, systemic and obstetrical examination was done paying special attention to the presence of risk factors for preterm labour. Gentle per speculum examination and if required a per vaginal examination and Bishop scoring was done. Complete haemogram, urine routine examination, urine culture and sensitivity, vaginal culture and sensitivity and CRP levels were done.

Ultrasonography was done as and when required to document fetal gestational parameters, rule out multiple pregnancy, congenital malformations and fetal growth restriction. Amniotic fluid index, placental localization and grading; cervical length and status of the cervical os were also assessed. Antibiotic was given according to culture and sensitivity report. These patients were followed up till delivery. Gestational age at the time of delivery, mode of delivery and neonatal outcome in terms of birth weight, morbidity and mortality were recorded.

Inclusion Criteria were duration of pregnancy between 28-37 weeks with uterine contraction of 4 in
20 minutes or 8 in 60 minutes each should last more than 20 seconds+ progressive changes in cervix, preterm premature rupture of membranes(leaking of amniotic fluid per vagina), cervical dilation $>1 \mathrm{~cm}$, cervical effacement of $80 \%$ or greater, UTI with labour pains after 28 weeks of gestation, chorioamnionitis, preterm labour due to polyhydramnious or multiple pregnancies. Exclusion Criteria were preterm pregnancy with intra uterine death.

After collection of data and editeding Data analysis was done by computer aided statistical software SPSS. The level of significance of 0.05 was used for the study.

\section{Results:}

Occurance: The total number of deliveries in the hospital of patients between 28-37 weeks were 1519 . Among them 210 patients presented in preterm labour giving frequency of preterm births of $13.82 \%$.

Table-I

Distribution of cases according to gestational age $(n=210)$

\begin{tabular}{|c|c|c|}
\hline Gestational Age & $\begin{array}{l}\text { No of } \\
\text { Cases }\end{array}$ & $\begin{array}{c}\text { Percentage }(\%) \\
(n-210)\end{array}$ \\
\hline $28-30$ weeks of gestation & 40 & $19.05 \%$ \\
\hline $31-33$ weeks of gestation & 71 & $33.81 \%$ \\
\hline $34-37$ weeks of gestation & 99 & $47.14 \%$ \\
\hline
\end{tabular}

Table-I shows maximum number of women(47.14\%) were in the gestational age group 34-37 weeks.

Table-II

Distribution of cases according to risk factor from past obstetric history.

\begin{tabular}{lcc}
\hline Risk Factor & $\begin{array}{c}\text { No. of } \\
\text { Cases }\end{array}$ & $\begin{array}{c}\text { Perecentage } \\
(\mathrm{n}-210)\end{array}$ \\
\hline Abortion & 46 & $21.90 \%$ \\
Preterm Delivery & 30 & $14.29 \%$ \\
Diabetes & 14 & $6.67 \%$ \\
Hypothyroidism & 3 & $1.43 \%$ \\
Anaemia & 55 & $26.19 \%$ \\
No risk factor found & 62 & $29.52 \%$ \\
\hline Total & 210 \\
\hline
\end{tabular}

Table-Il shows $21.9 \%$ had previous history of abortion and $14.3 \%$ has previous history of preterm delivery. 
Table-III

Distribution of cases according to risk factor in present pregnancy.

\begin{tabular}{lcc}
\hline Risk Factor & $\begin{array}{c}\text { No. of } \\
\text { Cases }\end{array}$ & $\begin{array}{c}\text { Perecentage } \\
(\mathrm{n}-210)\end{array}$ \\
\hline Vaginal Infection & 41 & $19.52 \%$ \\
UTI & 45 & $21.43 \%$ \\
PROM & 52 & $24.76 \%$ \\
PIH & 25 & $11.90 \%$ \\
Antepartum Haemorrhage & 24 & $11.43 \%$ \\
(APH) & & \\
Multiple Preganancy & 11 & $5.24 \%$ \\
Polyhydramnius & 12 & $5.71 \%$ \\
No risk factor & 31 & $14.76 \%$ \\
\hline Total & 241 \\
\hline
\end{tabular}

Table-III shows $24.8 \%$ cases of PROM, $21.4 \%$ cases of UTI and $19.5 \%$ cases vaginal infection associated with preterm labour.

Table-IV

Neonatal morbidity associated with prematurity(221 babies, Twin 11 cases)

\begin{tabular}{lcc}
\hline Neonatal Morbidity & $\begin{array}{r}\text { No. of } \\
\text { Cases }\end{array}$ & $\begin{array}{c}\text { Perecentage } \\
(\mathrm{n}-210)\end{array}$ \\
\hline Jaundice & 71 & $32.11 \%$ \\
RDS & 50 & $22.6 \%$ \\
Asphyxia & 30 & $13.57 \%$ \\
Sepsis & 23 & $10.40 \%$ \\
Hypoglycemia & 25 & $11.31 \%$ \\
Pneumonia & 13 & $5.88 \%$ \\
Intra Ventricular Haemorrhage & 9 & $4.07 \%$ \\
\hline Total & 221 \\
\hline
\end{tabular}

Table-IV shows that common morbidity of premature newborns were jaundice $32.11 \%$, RDS- $22.6 \%$ and asphyxia- $13.57 \%$

\section{Discussion:}

In our tertiary care centre the frequency of preterm births from period of June 2014 to December 2015 was $13.82 \%$. This is significantly more than the incidence of preterm birth given by researchers like Sumana and Misra et al; ${ }^{5}$ who reported a frequency of $10-12 \%$. The high occurance of preterm births in our institute is probably because, being a tertiary care centre dealing with high risk pregnancies, this includes those patients who delivered before term in view of other obstetric indications.
The maximum incidence was found between 34-37 weeks of gestation( $47.14 \%$ ) followed by $31-33$ weeks $(33.80 \%)$ and then between 28-30 weeks(19.04\%).

Risk factor of preterm labour was found to be multifactorial. We found a correlation between past obstetric history and the reproductive outcome in the present pregnancy; $21.9 \%$ patients presenting with preterm labour had a past history of abortions. Our results were consistent with the finding of Trivedi et al; ${ }^{6}$ Chhabra et al; ${ }^{7}$ and Singh et al $;{ }^{8}$ where $22.6 \%$, $18 \%$ and $14.4 \%$ subjects respectively had history of previous abortions. About fourteen percent patients in our study had history of preterm delivery in the previous pregnancies. Sigh Uma et al; ${ }^{9}$ also found that $14.4 \%$ patients had history of previous preterm delivery. Pandey et al; ${ }^{9}$ also concluded that prior preterm birth is a risk factor for preterm labour and it was identified in $14.4 \%$ subjects in their study group. Therefore, a detailed past obstetric history should be taken in all antenatal patients and those with a prior history of spontaneous abortion or preterm births should be counseled regarding the risk of preterm labour in the present pregnancy and managed accordingly.

In our study the commonest risk factor for preterm labour was preterm premature rupture of membranes which was associated with $24.8 \%$ preterm births. This is an accordance with Singh et al; ${ }^{8}$ study where preterm premature rupture of membranes was associated with $25.9 \%$ preterm births.

Another important cause of preterm labour in our study was genitourinary tract infection: $19.5 \%$ subjects in our study had vaginal infection. Singh et al; 8 found positive vaginal culture in $12.25 \%$ patients and Deka et al; ${ }^{10}$ found that cervical infection was present in as many as $55 \%$ patients with preterm labour; $21.4 \%$ patients in the study group had urinary tract infection. Similar finding were reported by pandey et al $;{ }^{9}$ Chhabra et al $;{ }^{7}$ and Singh et al $;{ }^{8}$ who found and incidence of $20.34 \%, 14 \%$ and $8.4 \%$ respectively confirming that UTI is an important factor for preterm labour. This means that it is important to diagnose UTI early and treat it aggressively to prevent preterm labour.

Antepartum haemorrhage contributed to $11.4 \%$ preterm birth in our study. Tocolysis was not offered to these patients and they were allowed to deliver. 
This is in accordance with the finding of Singh et al $;^{8}$ who also found that antepartum haemorrhage either led to spontaneous onset of preterm labour or induced labour in view of abruption placenta in $10.8 \%$ patients.

Multiple pregnancies were found to be associated with preterm labour in $5.2 \%$ of cases in our study. Arias et al; ${ }^{11}$ also reported that multiple pregnancy was responsible for $12-25 \%$ of all preterm deliveries.

The most common neonatal complications in our study group were jaundice(32.1\%), RDS(22.6\%) and Asphyxia(13.5\%). Sonkusare et $\mathrm{al}^{1}{ }^{12}$ reported the incidence of jaundice as $50.80 \%$, RDS as $20.16 \%$ and sepsis as $23.39 \%$. These were also the common complications in studies carried out by Singh et al; ${ }^{9}$ and Venket et al; ${ }^{13}$. The incidence of RDS was maximum in extremely premature babies and reduced significantly with increasing birth weight, gestational age $>34$ weeks and in patients who received two antenatal doses of injection Dexamethasone.

\section{Conclusion:}

This study concludes that the frequency of preterm birth from period of June 2014 to December 2015 was $13.82 \%$ in Monno medical College hospital,Manikganj. Following etiological factors were significantly associated with preterm birth i.e. previous history preterm delivery or abortion , premature rupture of membranes, anaemia, polyhydramnious, multiple pregnancies pregnancy induced hypertension.

The occurence of UTI and genital tract infection was more in patients presented with preterm labour. Neonatal morbidity and mortality was more in babies delivered prematurely and the most common cause of neonatal mortality among preterm infants was extreme prematurity and RDS.

Preventive measure may be taken nationally and institutionally to reduce prematurity and its consequences.

\section{References:}

1. Blencowe $\mathrm{H}$, Cousens $\mathrm{S}$, Oestergaard MZ et al. Data from national, regional and worldwide estimates of preterm birth rates in the year 2010. Obstetric Anesthesia Digest 2013;33(3):142.
2. Diallo FB, Diallo MS, Sylla M. et al;. Premature delivery- epidemiology, etiologic factors, prevention strategies. Dakar Med 1998; 43:70-3

3. Andrews K.M. Brouillette DB. Brouillette RT: "Mortality, Infant" Encyclopedia of Infant and Early Childhood Development. Elsevier 2008; 343-359

4. Uma S. Nisha S. Shikha S; A prospective analysis of etiology and outcome of preterm labour. Journal of Obstetrics and Gynecology India 2007; 57(1): 48-52

5. Sumama G, Misra R; preterm labour In: Ian Donald's Practical Obstetrical Problems $6^{\text {th }}$ edition(BI Publications Pvt Ltd. India) 2006; 397

6. Trivedi DR. Nagpal SP: preterm delivery: A common obstetric probem. Journal of Obstetrics and Gynecology of India 1995; 45: 380-384.

7. Chhabra S. Patil; Study of factors causing and arresting preterm labour. Journal of Obstetrics and Gynecology of India 2001; 51: 99-103

8. Singh U, Singh N, Seth S: A prospective analysis of etiology and outcome of preterm labour. Journal of Obstetrics and Gynaecology of india 2007; 57(1) 48-52

9. Pandey K, Bhagoliwal A, Gupta N, Geetanjaly $\mathrm{K}$ : Predictive value of various risk factors for preterm labour. Journal of Obstetrics and Gynecology of India 2010;60(2): 141-145

10. Deka D, Buckshec K, Kharsyntiew B, Gupta U: Association of Inter-amniotic fluid infection and cervical infection with preterm labour. Journal of Obstetrics and gynecology of india 1997;47:318-321

11. Arias F. Duftary SN, Bhide A: Preterm Parturition syndrome In: Practical Guide to High Risk Pregnancy and Delivery edited by Arias $\mathrm{F}$ $3^{\text {rd }}$ edition(Elsevier, USA) 2009:193

12. Sonkusare S, Rai I, Naik P; Preterm birth: Mode of delivery and neonatal outcome, Medical Journal of Malaysia 2009;64: 303-306

13. Venkat S. Nayyar R, Bhat J: Retrospective analysis of spontaneous $\mathrm{v} / \mathrm{s}$ induced preterm deliveries and neonatal outcome. Journal of Obstetrics and Gynecology of India; 2003;53: 46-48. 